\title{
Informer en temps d'épidémie: comment faire mieux
}

\author{
Arnaud Chioleroa, Stéphane Cullatib \\ a Professeur, MD, PhD; ${ }^{b}$ Dr, PhD - Laboratoire de santé des populations (\#PopHealthLab), Département de santé communautaire, Université de Fribourg
}

Quelles leçons tirer de la vague de Covid-19? Tenter un bilan sur le plan sanitaire est essentiel car une nouvelle vague est possible et on doit s'y préparer. Si le système de santé suisse a fait face à la crise de manière satisfaisante en comparaison internationale, la surveillance sanitaire et la communication sur le risque n'ont pas été convaincantes. Nous avons besoin de meilleures données mais il faut surtout savoir les rendre utiles pour la décision.

En Suisse, le système de santé semble, en comparaison internationale, avoir fait face de manière satisfaisante à cette crise, en s'adaptant en permanence pour assurer les soins nécessaires. Décentré, proche des besoins de la population, il a démontré sa souplesse et sa résilience; les professionnels de santé et les administrations impliquées dans cette crise ont fait preuve d'un engagement impressionnant, de professionnalisme et de solidarité. On aurait du bien sûr mieux se préparer, en particulier hors des hôpitaux où les prestataires de soins (médecins installés, pharmaciens, CMS et EMS) ont été insuffisamment soutenus et trop peu intégrés dans les stratégies de gestion de cette épidémie. Les coûts ont été importants, et il y a une souffrance et une fatigue des professionnels de santé dont il faut s'occuper.

Nettement moins convaincantes auront été la surveillance et la communication sur le risque sanitaire.

\section{Pléthore de données, peu d'info utile}

Une surveillance sanitaire utile est celle qui permet de produire de l'information qui aide à la décision (fig. 1). Il ne suffit pas d'analyser des données et de les publier; il faut être attentif à comment cette information est disséminée et aux modalités de communication auprès des responsables de santé de publique ou de la population, et c'est un métier [1].

Avec la Covid-19, les données n'ont pas manqué; on a même été submergé par des données, accessibles - en open access - comme jamais auparavant. De ces données ont émergés beaucoup d'analyses statistiques, beaucoup de courbes, de prévisions et beaucoup de bruit dans les médias et sur les réseaux sociaux; il en a résulté une «infodémie» mais étonnamment peu d'information utile pour la décision. Comment expliquer cela?

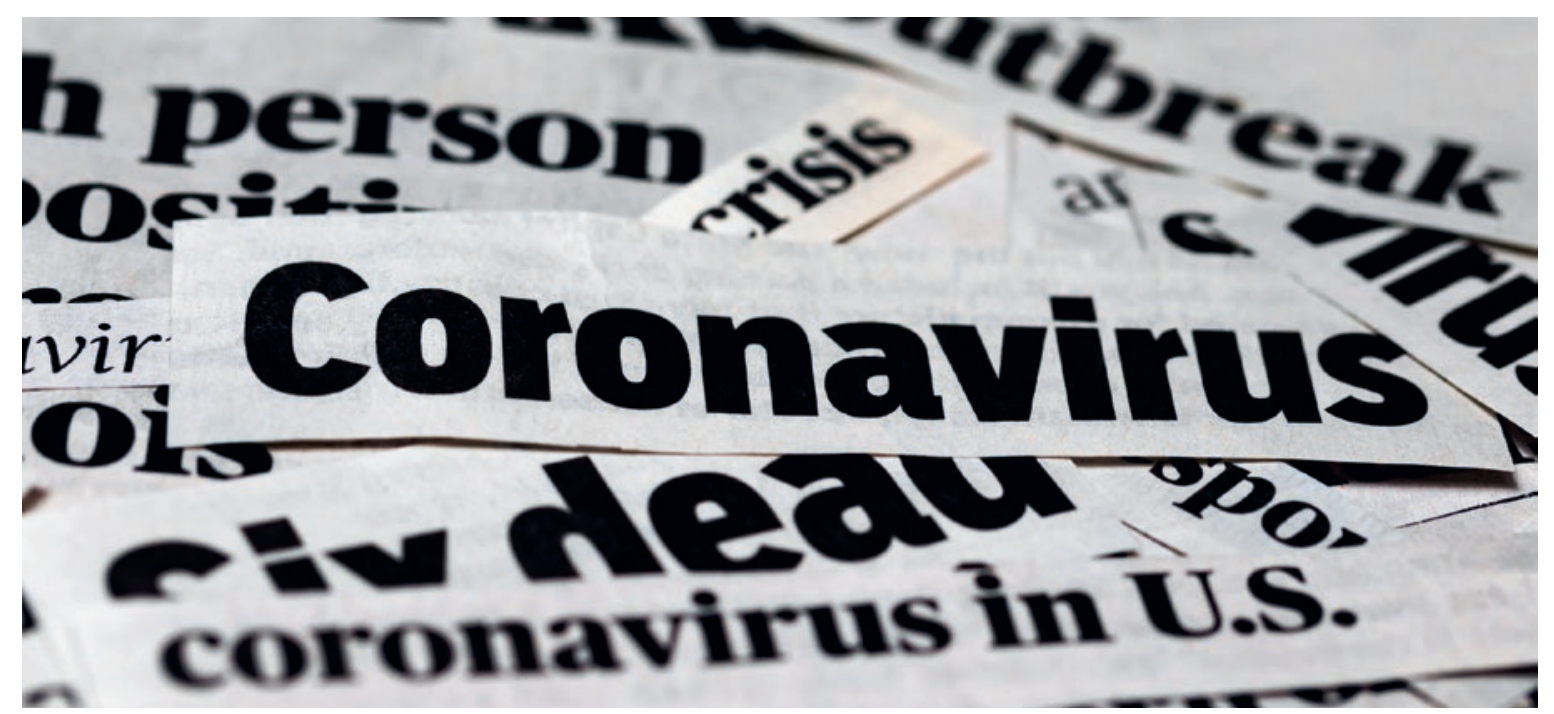

Annonces officielles, médias, scénarios catastrophe: la population a rarement été autant submergée par les données que pendant la crise du coronavirus. (Image symbolique: (c) Jj Gouin | Dreamstime.com) 
La faible qualité des données est bien entendu en cause mais le problème majeur, révélé douloureusement par cette crise, est la confusion entre la surveillance sanitaire et la recherche épidémiologique. Ces domaines se nourrissent des mêmes données mais ont des finalités différentes: la surveillance vise à produire de l'information utile pour la décision en santé publique alors que la recherche en épidémiologie vise à produire de la connaissance (fig. 2).

De nombreux épidémiologistes spécialistes dans l'analyse de données assument que la connaissance tirée de leurs analyses suffit pour informer. D'une part, ces chercheurs ne mesurent pas toujours les faiblesses des données auxquelles ils ont accès. Déconnectés des prestataires de soins, ils ne comprennent pas toujours comment les données sont produites et ce qui peut fondamentalement les biaiser.

D'autre part, même en tenant compte de la faiblesse des données, il est difficile de communiquer sur le risque sanitaire en particulier lorsque l'incertitude est grande. Beaucoup de scientifiques ne savent pas comment s'adresser aux responsables de santé publique ou à la population. Car savoir ne suffit pas. Annoncer via les médias jusqu'à 30000 morts en Suisse ou que les lits de soins intensifs vont manquer, alors que l'incertitude est énorme, génère de la peur sans donner une information vraiment utile pour la décision. Alors que ces prévisions sont avant tout des simulations pour définir des scénarios (parfois extrêmes) de planification sanitaire, elles sont trop souvent comprises comme «c'est ce qu'il va arriver»; l'incertitude n'est pas suffisamment clairement communiquée [2].

\section{Riche en données et en informations}

On doit passer d'un système riche en données mais pauvre en informations à un système riche en données et en informations [3]. Alors que pour les soins la cen-

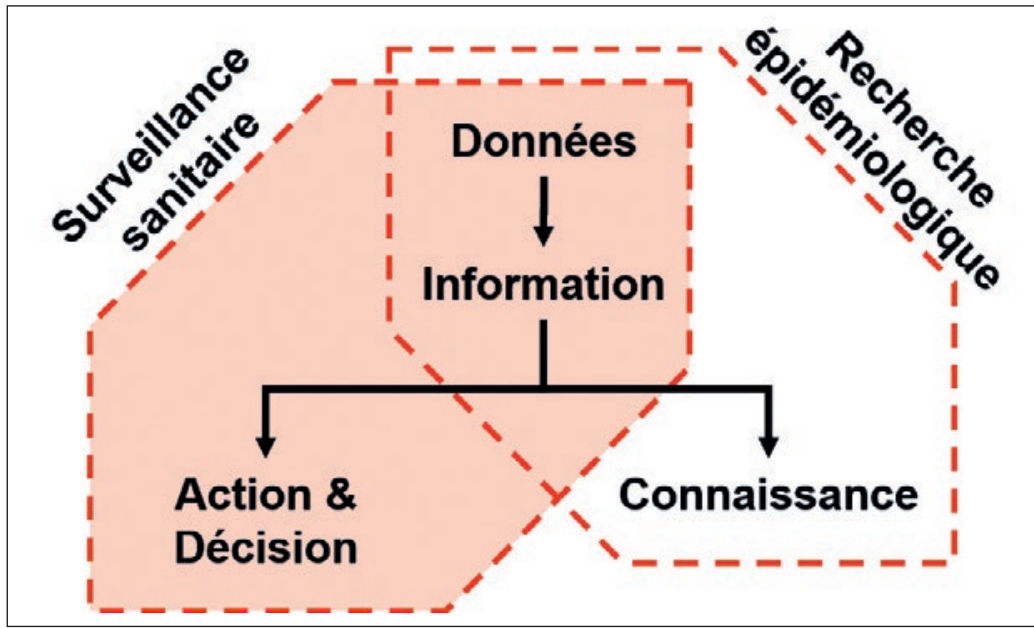

Figure 2: Distinction entre surveillance sanitaire et recherche épidémiologique [1].

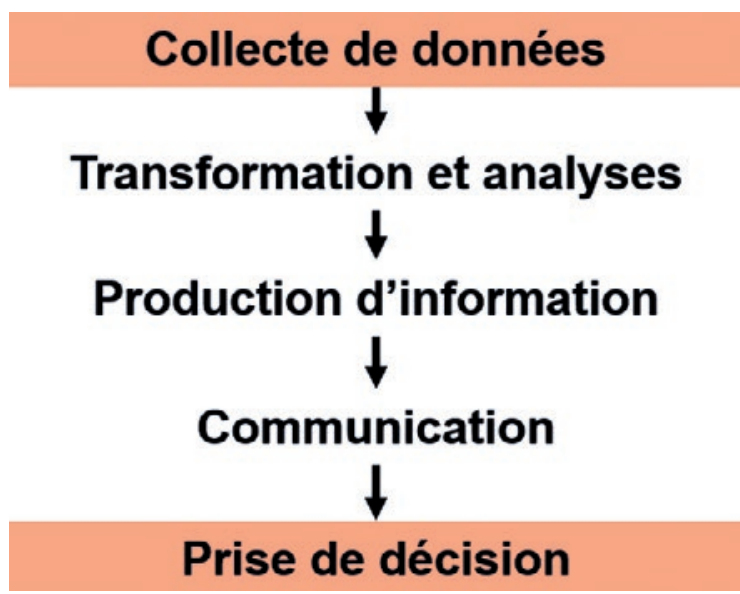

Figure 1: Etapes dans le traitement des données pour la surveillance sanitaire, de la collecte de données à la prise de décision [1].

tralisation n'est certainement pas pertinente, elle est nécessaire pour une surveillance efficace. Entre les prestataires de soins, et entre les cantons, il faut standardiser les collectes de données, notamment sur les tests, les cas et les décès, et ce de manière pérenne, avec comme objectif une analyse exhaustive et en temps réel. Dans le domaine des hôpitaux, un registre national exemplaire est celui des lits des soins intensifs qui s'est constitué sous la pression de l'épidémie [4].

Le dossier électronique partagé (DEP) a aussi le potentiel à terme de faciliter le monitoring de l'activité ambulatoire lors de pandémie [5].

Enfin, il faut surtout mieux communiquer. Les hautes écoles et les chercheurs spécialistes des données ont démontré leur puissance d'analyse mais aussi leur peine à produire des informations utiles à la décision en santé publique. Pour faire le lien entre les scientifiques qui analysent des données et les décideurs, il faut des experts en transfert de connaissance proches des organes de décisions [6, 7]. Les données ne parlant pas toutes seules, il faut les rendre utiles.

\section{De la météo aux épidémies}

Prenons l'exemple de la météorologie, dont on peut s'inspirer [2,8] pour améliorer l'utilité des prévisions épidémiologiques. Alors que les prévisions météorologiques sont aussi le produit de modèles extrêmement complexes et soumis à d'importantes incertitudes, elles sont utilisées quotidiennement pour se préparer à des dangers, tels des tempêtes ou canicules. Mais on accepte l'incertitude inhérente à ces prévisions. De même, les hypothèses complexes des modèles épidémiologiques et l'incertitude qui en découle doivent être assumées [2]; il n'y a pas une prévision - elles sont toujours multiples. La communication sur ces prévi- 
sions doit aussi tenir compte du contexte sanitaire global; c'est de la responsabilité civique et éthique des scientifiques d'être attentifs aux effets psychologiques, sociétaux et politiques de leurs annonces.

Enfin, et par analogie avec MétéoSuisse, un organisme national pour coordonner ces activités de surveillance, prévision et communication est certainement nécessaire. Technique et apolitique, et travaillant étroitement avec les prestataires de soins, les assurances, les experts de la santé publique et les hautes écoles, un tel organisme serait le garant d'une surveillance plus efficace et véritablement au service des cantons, de la Confédération et de la population.

\section{Crédits images}

Graphiques: Arnaud Chiolero

\section{Références}

1 Chiolero A, Buckeridge D. Glossary for public health surveillance in the age of data science. J Epidemiol Community Health. 2020; jech-2018-211654.

2 Saltelli, et al. Five ways to ensure that models serve society: a manifesto. Nature. 2020;582:482-4.

3 OECD. Health in the 21st century: putting data to work for stronger health systems. Paris: Organisation for Economic Co-operation and Development, 2019.

4 Zhao C, et al. icumonitoring.ch: a platform for short-term forecasting of intensive care unit occupancy during the COVID-19 epidemic in Switzerland. Swiss Med Wkly. 2020;150:w20277.

5 Gilli Y. La numérisation, grande gagnante de la crise. Et le DEP? Bull Med Suisses. 2020;101(25-26):783.

6 Swiss School of Public Health https://ssphplus.ch/en/covid-19/, accédé le 18.6.2020.

7 Quinto C. Manque d'expertise aux postes décisifs. Bull Med Suisses. 2020;101(25-26):817.

8 Rivers C, George D. How to Forecast Outbreaks and Pandemics. www.foreignaffairs.com/articles/united-states/2020-06-29/ how-forecast-outbreaks-and-pandemics, accédé le 1.7.2020.
Pr Arnaud Chiolero, MD PhD Population Health Laboratory (\#PopHealthLab) Département de santé communautaire Université de Fribourg Route des Arsenaux 41 $\mathrm{CH}-1700$ Fribourg arnaud.chiolero[at]unifr.ch

\section{L'essentiel en bref}

- Pendant la crise du Covid-19, la population a croulé sous les données. L'«infodémie» qui en a résulté ne contenait que peu d'information utile pour la décision.

- A la source du problème, la confusion entre la surveillance sanitaire, visant à produire de l'information utile pour la décision en santé publique, et la recherche épidémiologique, qui crée de la connaissance.

- Les scientifiques ne mesurent pas toujours les faiblesses des données et ignorent souvent comment informer le grand public. Or, communiquer sur le risque sanitaire est d'autant plus difficile que l'incertitude est grande. Les prévisions sont trop souvent comprises comme des prédictions et non comme des simulations. L'incertitude doit être plus clairement communiquée.

- Les auteurs plaident pour un système à la fois riche en données et en informations. Pour disposer d'une surveillance efficace, il faut centraliser les collectes de données (tests effectués, cas, décès) et surtout mieux les communiquer. Cette tâche doit revenir à des experts pouvant faire le lien entre scientifiques et décideurs.

\section{Das Wichtigste in Kürze}

- Während der COVID-19-Krise wurde die Bevölkerung von Informationen überschwemmt. Die «Infodemie» enthielt wenig nützliche Informationen für die Entscheidungsfindung.

- Das Problem: Gesundheitsüberwachung, welche für die Entscheidungsfindung im Bereich der öffentlichen $\mathrm{Ge}$ sundheit nützliche Informationen zu produzieren soll, und epidemiologische Forschung, die Wissen schafft, wurden nicht klar getrennt.

- Forschende sind sich der Datenschwächen und wie man die Öffentlichkeit informiert nicht immer bewusst. Je größer die Unsicherheit, desto schwieriger ist es, Gesundheitsrisiken zu kommunizieren. Prognosen werden allzu oft eher als Voraussagen denn als Simulationen verstanden. Die Unsicherheit muss klarer kommuniziert werden.

- Die Autoren plädieren für eine wirksame Überwachung welche eine zentralisierte Datenerfassung erfordert (durchgeführte Tests, positive Fälle, Todesfälle), und vor allem für bessere Kommunikation. Diese Aufgabe muss von Ex perten wahrgenommen werden, die als Bindeglied zwischen Wissenschaftlern und Entscheidungsträgern fungieren. 